\title{
Rimonabant for overweight and "metabolic syndrome": the attempt to supersize disease and risk by pharmaceutical marketing
}

\author{
Rita Banzi · Lorenzo Moja · Ivan Moschetti · \\ Alessandro Liberati · Gian Franco Gensini · \\ Roberto Gusinu · Andrea A. Conti
}

Received: 8 February 2008/Accepted: 8 February 2008/Published online: 4 March 2008

(C) SIMI 2008

\section{The methodologist's point of view}

Rita Banzi · Lorenzo Moja · Ivan Moschetti ·

Alessandro Liberati

A Cochrane systematic review explored the potential role of rimonabant for overweight and obesity [1]. On the basis of surrogate outcomes reported in the included RCTs, rimonabant has been a candidate (by its producer) to act as a pleiotropic agent for the entire cardiovascular risk spectrum. Is this compelling evidence to consider rimonabant the new panacea for the "metabolic syndrome" or are we facing another attempt of disease mongering by a new market frontier?

Rimonabant has been shown to reduce food intake, appetite and body weight in overweight or obese people. Four randomized controlled trials (RIO-Europe [2], RIONorth America [3], RIO-diabetes [4], RIO-lipids [5])

R. Banzi · L. Moja ( $₫)$ I. Moschetti · A. Liberati Italian Cochrane Centre, Mario Negri Institute for Pharmacological Research, Via Eritrea 62, 20157 Milan, Italy

e-mail:moja@marionegri.it

G. F. Gensini

Department of Critical Care Medicine and Surgery,

University of Florence and Azienda Ospedaliero-Universitaria Careggi, Florence, Italy

R. Gusinu

DAI Cardiologico e dei Vasi, Azienda Ospedaliero-Universitaria Careggi, Florence, Italy

A. A. Conti

Department of Critical Care Medicine and Surgery,

University of Florence and Don Carlo Gnocchi Foundation

IRCCS, Florence, Italy evaluated rimonabant $20 \mathrm{mg}$ versus rimonabant $5 \mathrm{mg}$ versus placebo. All interventions were given over 1 year and included the addition of a hypocaloric diet. All trials were designed, conducted and reported with the contribution of Sanofi-Aventis drug company. The Cochrane review ("Rimonabant for overweight and obesity") [1] includes evidence from these four trials.

Cochrane review

Overall 6,625 patients aged 18 and above, with a BMI $\geq 27 \mathrm{~kg} / \mathrm{m}$ and with treated or untreated hypertension or dyslipidaemia were enrolled. Specific inclusion criteria for RIO-Diabetes and RIO-Lipids were uncontrolled type 2 diabetes and untreated dyslipidaemia, respectively. In all trials the primary outcome was the change in weight from baseline to 1 year post-treatment. Among secondary outcomes, the prevalence of metabolic syndrome after 1 year and the change in cardiometabolic risk factors, such as blood pressure and lipid profile, were evaluated.

Compared to placebo, the meta-analysis of the included studies showed a statistically significant weight reduction with a mean of $4.64 \mathrm{~kg}$ after 1 year for rimonabant $20 \mathrm{mg}$, while the pooled effect of rimonabant $5 \mathrm{mg}$ was a weight reduction of $1.25 \mathrm{~kg}$ after 1 year, resulting in a very limited clinical effect. Improvement in waist circumference, highdensity lipoprotein cholesterol, trygliceride levels and blood pressure were also observed with patients on rimonabant $20 \mathrm{mg}$.

Patients treated with rimonabant $20 \mathrm{mg}$ reported significantly more serious adverse events $(\mathrm{RR}=1.37,95 \%$ CI 1.04, 1.80) and greater rate of discontinuation due to adverse effects $(\mathrm{RR}=1.92,95 \%$ CI $1.57,2.34)$ than placebo. The main safety issues were adverse psychiatric events, especially because patients with current depressions 
were excluded from the clinical trials. Even in this highly selected population anxiety, mood disorders and depressive symptoms were reported more frequently with rimonabant $20 \mathrm{mg}$ in comparison to rimonabant $5 \mathrm{mg}$ and placebo.

\section{Cochrane review as drug company amplifier?}

Individual studies included in this review were all sponsored by the producer of rimonabant; this could have led to a conflict of interest which could have affected the results. There is evidence that Pharma companies' sponsored studies tend to provide more often positive results and overestimate treatment effects [6-8]. Publication and outcome reporting biases cannot be ruled out, as preliminary trials have probably been done but are not retrievable. The high attrition rate (i.e. patient losses during the studies) in both treatment and placebo groups (approximately $40 \%$ in all studies), which is difficult to compensate by any form of analysis, had lowered the quality of the studies. One could have expected a more thorough critical appraisal of the clinical relevance of the evidence from the Cochrane reviewers; to avoid that meta-analysis could become an amplifier of non-pertinent and irrelevant results, eventually pushing the drug's promotion.

\section{Rimonabant in the context}

As a general guideline, the US National Institutes of Health (NIH) recommended that the initial goal of weight loss therapy in overweight and obese patients should be to reduce body weight by approximately $10 \%$ from baseline [9]. Following this indication, both the European Medicines Agency (EMEA) and the American Food and Drug Administration (FDA) require any anti-obesity agent to produce a weight loss of $10 \%$ of initial bodyweight compared to placebo [10]. The use of rimonabant after 1 year of treatment produces a modest weight loss of approximately $5 \%$. Comparing this result with two other approved anti-obesity drugs, orlistat and sibutramine, it could be argued that the weight loss associated with rimonabant is slightly greater than orlistat and comparable to sibutramine [11]. These comparisons are indirect and readers should be aware that there are often systematic differences between the trials addressing one intervention and the trials addressing the other, taking the results of these comparisons cautiously. In 2006 Rimonabant 20 mg film-coated tablets had been approved, on the basis of the results of these four RCTs, by the EMEA under the trade name Acomplia. The approved indication is "as an adjunct to diet and exercise for the treatment of obese patients $\left(B M I \geq 30 \mathrm{~kg} / \mathrm{m}^{2}\right.$ ) or overweight patients (BMI $\geq 27 \mathrm{~kg}$ / $\mathrm{m}^{2}$ ) with associated risk factor(s), such as type 2 diabetes or "dyslipidaemia" [12].
The attempt to expand the market for rimonabant

Beyond weight management, the proposed indications by the producer included treatment for type 2 diabetes, dyslipidaemia, smoking cessation, and management of multiple cardiometabolic risk factors in patients with metabolic syndrome. Although rimonabant has shown potential in the management of these conditions, the lack of comparative data with standard therapies and/or the uncertainties regarding the consistency and magnitude of the effect (i.e. smoke cessation) were the bases for a negative benefit risk assessment by the EMEA. Although relevant decreases in certain cardiac risk factors associated with obesity have been seen with a loss of at least $5-10 \%$ of initial weight, information regarding the effect of rimonabant on major cardiovascular events will only be available when the results of the CRESCENDO trial, which looks to these events in more than 17,000 patients treated with rimonabant for 5 years, are finalized in a few years [13].

An arbitrary and fluid outcome: the metabolic syndrome

Within the EMEA regulatory process, the "metabolic syndrome indication" required a profound discussion by an adhoc panel of experts, who agreed that metabolic syndrome cannot be considered today as a target for therapy in itself, but rather as an additional tool to identify patients at high cardiometabolic risk. The term "metabolic syndrome" refers to a clustering of specific cardiovascular disease (CVD) risk factors whose underlying pathophysiology is believed to be related to insulin resistance. It has been postulated that insulin resistance and its compensatory hyperinsulinemia predisposes patients to hypertension, hyperlipidaemia, diabetes, and obesity [14]. The available definitions of the metabolic syndrome for diagnostic purposes need for further harmonisation, as different organisations have developed similar but not identical criteria, excluding other risk factors such as age and history of CVD events. Current definitions of the syndrome capture people with diagnosed disease (e.g. diabetes, hypertension, clinical CVD), as well as those who have milder and "normal" conditions. Although some authors stated that in the near future new definition(s) will be applicable worldwide [15], others suggested that this expansion in prevalence estimates exploits the widespread profitable short- and long-term use of rimonabant, creating a new market [16].

In light of these considerations, it should be assessed if and how the management of the metabolic syndrome as a whole would differ from the treatment of the individual components. There is no question that the risk factors for cardiovascular disease cluster together. The question is 
whether the cluster of features conceals a distinct clinical entity. In other words, it is far to be fully demonstrated that the whole is greater than the sum of the parts. In the near future, the risk would be that rimonabant will be proposed to any one individual overweight or with just a threat of cardiovascular symptoms. A broad population, with the largest majority of subject at low-baseline risk of disease, will be exposed to medicalization and adverse events without knowing the real benefits deriving from this treatment [17]. The outcome being probabilistic, CVD events will not be prevented because they will never develop. For a public health system the related costs will be difficult to absorb. This strategy has been defined as disease mongering, i.e. the selling of sickness that widens the boundaries of illness and grows the markets for those who sell and deliver treatments. [18] We invite readers to take a careful look at a recent special issue of PLoS Medicine-a leading open access journal-to have a proper understanding of this phenomenon [17-19].

The "rimonabant regulatory case" constitutes a very important precedent in the field of cardiovascular drugs. Naming a syndrome does not automatically mean that it exists. In the field of cardiovascular risk factors the temptation to enlarge the "abnormal" population and, subsequently, the market of treatment is very high. An "universal" indication as "Management of multiple cardiovascular risk factors in patients with metabolic syndrome", which will expose millions of patients to a drug therapy, cannot be granted on the basis of the very limited knowledge of the metabolic syndrome available to date. Furthermore, assuming the validity of the metabolic syndrome definition, the lack of comparative data with the existing cardiovascular risk factor treatments (statins, antihypertensive agents, hypoglicaemic agents, physical exercise, etc.) precludes any attempt to find rimonabant a "place in therapy". It should be underlined that new clinical and therapeutical options should address relevant public health needs rather than market-driven pseudoneeds. To set new drug indications into a more relevant scenario, clinicians, reviewers and regulatory agencies should always consider to base their evaluation on the body of evidence already available, being resistant to market drift.

\section{A clinician's point of view}

\section{Gian Franco Gensini · Roberto Gusinu •}

Andrea A. Conti

In the past few years the popularity of the "metabolic syndrome" has become more and more diffused, paralleling the increasing discussion relative to its epidemiological definition, pathophysiological meaning, and clinical relevance. The metabolic syndrome is an articulated clinical complex interpreted as a clustering of risk factors associated with an increased risk in cardiovascular diseases and mortality and in diabetes mellitus. In some studies, its prevalence in the USA has been computed as being even more than $25 \%$ in the population [20].

On diagnostic grounds, various definitions for the "metabolic syndrome" have been proposed through time; in 1999, for example, the World Health Organization stated that, to make the diagnosis of this syndrome, a patient should present with impaired glucose tolerance, impaired fasting glucose, insulin resistance or diabetes mellitus, plus two or more of the following elements: arterial hypertension, central obesity, increased plasma triglycerides and/or low HDL cholesterol, microalbuminuria [21].

According to the new International Diabetes Federation definition (2005), central obesity has become the pivotal factor, and to perform the diagnosis, obesity must be accompanied by at least two of the following four elements: reduced HDL cholesterol or specific therapy for this disorder, increased triglycerides values or specific therapy for this disorder, arterial hypertension or therapy of previously diagnosed hypertension, raised fasting plasma glucose or previously diagnosed type 2 diabetes mellitus [21].

The different definitions available on the one hand indicate that a general consensus on the "metabolic syndrome" seems to have been not yet achieved, and, on the other, point to the fact that, in any case, the therapeutic management of this syndrome should be harmonic and integrated.

Rimonabant has only one therapeutic target (body weight) and therefore, from a theoretical point of view, only concentrates on a specific element, and not on the whole complex clinical entity called "metabolic syndrome". From an operational standpoint, indirect comparisons with other approved drugs for obesity, such as sibutramine and orlistat, show that the weight loss associated with rimonabant overlaps that with sibutramine and it is slightly higher than that with orlistat. Considering also the depressive symptoms and mood disorders reported by patients on rimonabant in clinical trials (as indicated in the meta-analysis by Christensen et al. [22]), the efficacy and safety profiles of this drug consequently need further structured systematic evaluation.

This appears particularly relevant today, in an era of evidence-based medicine, so as to vigorously prevent and reduce the so-called "disease mongering" phenomenon [23]. Since various clinical elements associate in a synergic negative congregation in subjects at increased risk of cardiovascular disease, evidence-based therapeutic interventions should contemporaneously target different 
features, having clearly demonstrated their efficacy and safety on the combination of the different elements of the so-called "metabolic syndrome", and therefore on the patient/person as a whole.

\section{References}

1. Curioni C, Andre C (2006) Rimonabant for overweight or obesity. Cochrane Database Syst Rev, CD006162

2. Van Gaal LF, Rissanen AM, Scheen AJ, Ziegler O, Rossner S (2005) Effects of the cannabinoid-1 receptor blocker rimonabant on weight reduction and cardiovascular risk factors in overweight patients: 1-year experience from the RIO-Europe study. Lancet 365:1389-1397

3. Pi-Sunyer FX, Aronne LJ, Heshmati HM, Devin J, Rosenstock J (2006) Effect of rimonabant, a cannabinoid-1 receptor blocker, on weight and cardiometabolic risk factors in overweight or obese patients: RIO-North America: a randomized controlled trial. JAMA 295:761-775

4. Scheen AJ, Finer N, Hollander P, Jensen MD, Van Gaal LF (2006) Efficacy and tolerability of rimonabant in overweight or obese patients with type 2 diabetes: a randomized controlled study. Lancet 368:1660-1672

5. Despres JP, Golay A, Sjostrom L (2005) Effects of rimonabant on metabolic risk factors in overweight patients with dyslipidemia. N Engl J Med 353:2121-2134

6. Bero L, Oostvogel F, Bacchetti P, Lee K (2007) Factors associated with findings of published trials of drug-drug comparisons: why some statins appear more efficacious than others. PLoS Med 4:e184

7. Djulbegovic B et al (2000) The uncertainty principle and industry-sponsored research. Lancet 356:635-638

8. Melander H, Ahlqvist-Rastad J, Meijer G, Beermann B (2003) Evidence $b(i)$ ased medicine-selective reporting from studies sponsored by pharmaceutical industry: review of studies in new drug applications. BMJ 326:1171-1173
9. NHI Clinical guidelines on the identification, evaluation, and treatment of overweight and obesity in adults. http://www.nhlbi. nih.gov/guidelines/obesity/ob_home.htm

10. EMEA (2006) Guideline on clinical investigation of medicinal product used in weight control. http://www.emea.europa.eu/pdfs/ human/ewp/028196en.pdf

11. Rucker D, Padwal R, Li SK, Curioni C, Lau DC (2007) Long term pharmacotherapy for obesity and overweight: updated metaanalysis. BMJ 335:1194-1199

12. EMEA European Public Assessment Report (EPAR). http://www. emea.europa.eu/humandocs/Humans/EPAR/acomplia/acomplia. htm. Last accessed Jan 2007

13. CRESCENDO Trial. http://clinicaltrials.gov/show/NCT00263042

14. Reaven GM, Chen YD (1988) Role of insulin in regulation of lipoprotein metabolism in diabetes. Diabetes Metab Rev 4:639652

15. Eckel RH, Grundy SM, Zimmet PZ (2005) The metabolic syndrome. Lancet 365:1415-1428

16. Gale EA (2005) The myth of the metabolic syndrome. Diabetologia 48:1679-1683

17. Heath I (2006) Combating disease mongering: daunting but nonetheless essential. PLoS Med 3:e146

18. Moynihan R, Henry D (2006) The fight against disease mongering: generating knowledge for action. PLoS Med 3:e191

19. Mintzes B (2006) Disease mongering in drug promotion: do governments have a regulatory role? PLoS Med 3:e198

20. Eckel RH, Grundy SM, Zimmet PZ (2005) The metabolic syndrome. Lancet 365:1415-28

21. Zimmet PZ, Alberti G (2005) Medscape diabetes \& endocrinology. 7(2). Available at http://www.medscape.com/viewarticle/ 514211. Accessed on January 31, 2008

22. Christensen R, Kristensen PK, Bartels EM, Bliddal H, Astrup A (2007) Efficacy and safety of the weight-loss drug rimonabant: a meta-analysis of randomized trials. Lancet 370:1706-1713

23. Moynihan R, Heath I, Henry D (2002) Selling sickness: the pharmaceutical industry and disease mongering. BMJ 324:886-891 